\title{
Characterization of a 300-GHz Transmission System for Digital Communications
}

\author{
Martin Hudlička ${ }^{1}$ (D) Mohammed Salhi' ${ }^{2}$. \\ Thomas Kleine-Ostmann ${ }^{2}$ - Thorsten Schrader ${ }^{2}$
}

Received: 13 December 2016 / Accepted: 7 May 2017/

Published online: 17 May 2017

(C) The Author(s) 2017. This article is an open access publication

\begin{abstract}
The paper presents the characterization of a 300-GHz transmission system for modern digital communications. The quality of the modulated signal at the output of the system (error vector magnitude, EVM) is measured using a vector signal analyzer. A method using a digital real-time oscilloscope and consecutive mathematical processing in a computer is shown for analysis of signals with bandwidths exceeding that of state-of-the-art vector signal analyzers. The uncertainty of EVM measured using the real-time oscilloscope is open to analysis. Behaviour of the $300-\mathrm{GHz}$ transmission system is studied with respect to various modulation schemes and different signal symbol rates.
\end{abstract}

Keywords Error vector magnitude $\cdot$ Waveform metrology $\cdot \mathrm{THz}$ communication

\section{Introduction}

Nowadays, spectral resources are very limited as new communication technologies providing higher data rates are being developed. The frequencies are shifting towards higher unoccupied bands in the millimetre and submillimeter wave range. Millimeter-wave communication systems mature [1-3] and the focus of research is, naturally, moving up to the $\mathrm{THz}$ range. Prospective $\mathrm{THz}$ communication systems

Martin Hudlička

mhudlicka@cmi.cz

1 Czech Metrology Institute, Okružní 31, 63800 Brno, Czech Republic

2 Physikalisch-Technische Bundesanstalt, Bundesallee 100, 38116 Braunschweig, Germany 
are studied, e.g. papers [4-6] and references therein. Recently, several experiments demonstrated a successful transmission at frequencies of 60 to $400 \mathrm{GHz}$ [7-11]. Although THz technology could satisfy the demand for an extremely high data rate, a number of technical challenges need to be overcome or better understood before its development.

Recently, a transmission system has been developed [12] which aims at the metrological characterization of propagation channels, material properties and field strengths in the $300-\mathrm{GHz}$ frequency band. Several communication experiments have been performed $[12,13]$. The error vector magnitude (EVM), which is the key qualitative parameter used in wireless communication, has been measured using a vector signal analyzer (VSA). Parameters such as EVM are not easily traceable to basic physical quantities (traceability means an unbroken chain of comparisons relating an instrument specific measurement to a known standard), and thus often, the measurement accuracy is questionable.

In this paper, we aim to test the transmission system [12] usability for digital communications. The quality of the signal (EVM) will be measured at the output of the receiver. Approaches for traceable measurement of EVM have been shown recently. The British National Physical Laboratory uses waveform metrology techniques based on digital real-time oscilloscopes (DRTO) and digital sampling oscilloscopes (DSO) that form an unbroken chain between the electro-optic sampling system and the modulated RF waveform. Traceability is available for wideband RF power (GSM and other formats) [14] and techniques for wideband code division multiple access (WCDMA) have been presented [15, 16]. A procedure for measurement of the EVM of a source and receiver separately using a DRTO is given in [17].

The high bandwidth of state-of-the-art DRTOs makes them an ideal tool for characterization of future ultrawideband communication systems as shown, e.g. in [19], where a signal with a bandwidth of $1.76 \mathrm{GHz}$ has been captured using a $32-\mathrm{GHz}$ bandwidth oscilloscope with vector signal analysis software. The bandwidth of modern VSAs does not exceed $500 \mathrm{MHz}$. On the other hand, they are characterized by much higher vertical resolution (typically $>12$ bits) in comparison to high-bandwidth DRTOs (vertical resolution typically $<8$ bits). Traceability for wideband real-time oscilloscopes is discussed in [18]. The transmission system developed in [12] is able to transmit a signal bandwidth of $10 \mathrm{GHz}$. Such a bandwidth cannot be analyzed with use of any VSA commercially available today. Basic measurements of the EVM in the $300-\mathrm{GHz}$ transmission system using a DRTO and subsequent processing of measured samples using in-house code have been performed in [20]. In this paper, more thorough analysis of the measurement system is given and more modulation schemes are considered. Some hardware issues are discussed and simple measurement uncertainty analysis is carried out.

The remainder of this paper is structured as follows. In Section 2, calculation of EVM and several related technical aspects are introduced. In Section 3, the transmission system setup is described. Section 4 shows EVM measurements for several scenarios. The EVM measurement uncertainty analysis is discussed in Section 5. Finally, in Section 6, we conclude and give an outlook and general remarks to the choice of a suitable modulation scheme for multigigabit transmissions. 


\section{Error Vector Magnitude Evaluation}

The EVM is one of the parameters used to define the performance of wireless communication equipment. It is defined as the magnitude of the difference between the reference waveform and the measured waveform in a vector diagram (see Fig. 1). A signal sent by an ideal transmitter or captured by a receiver would have all points of the constellation diagram precisely at ideal locations. However, various imperfections in the implementation (such as carrier leakage, low image rejection ratio and phase noise) cause the actual constellation points to deviate from the ideal locations. Prior to EVM calculation, the received signal has to be corrected (RF frequency offset, timing offset, phase offset etc.) in order to minimise the error vector (see, e.g. [21]). The EVM is given by

$$
\mathrm{EVM}_{\mathrm{RMS}}=\sqrt{\frac{\frac{1}{N} \sum_{n=1}^{N}\left|e_{\mathbf{x}}[n]\right|^{2}}{\frac{1}{N} \sum_{n=1}^{N}|r[n]|^{2}}},
$$

where

$$
\begin{aligned}
e_{\mathbf{x}}[n]= & \left(x_{1}+j x_{2}\right) t[n] \exp \left[-\left(x_{3}+j \kappa x_{4}\right)(n-1)\right] \\
& -\left(x_{5}+j x_{6}\right)-r[n], \quad n=1, \ldots, N
\end{aligned}
$$

is the model obtained for a residual complex error when the compensations are included [22]. The $\kappa=2 \pi T_{\text {symb }}$ is the sampling period, $\left(x_{1}+j x_{2}\right)$ is the complex gain factor, $x_{3}$ is the loss factor, $x_{4}$ is the frequency offset and $\left(x_{5}+j x_{6}\right)$ is the complex origin offset. The reference signal is represented by $r[n]$ and subscript $\mathbf{x}$ represents the vector of real parameters $x_{1}, \ldots, x_{6}$. Manufacturers of measurement instrumentation have usually developed their own signal processing methods to modify the signal prior to EVM calculation, and these methods are neither publicly available nor comparable among different vendors. This is one of the reasons why no manufacturer is able to measure the modulated signal parameters traceably, yet. The situation is even more complicated in fourth-generation $(4 \mathrm{G})$ systems, where the EVM definition is problematic (see, e.g. [23]). Additional practical hints for calculation of the EVM can be found (e.g. in [24]).

Fig. 1 Definition of EVM in a constellation diagram

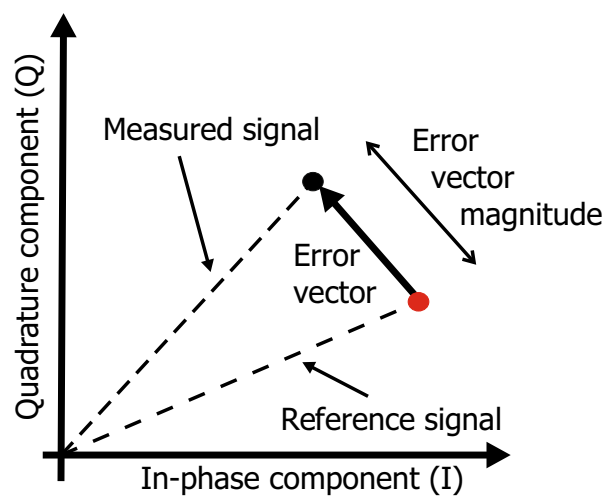




\section{System Setup}

A detailed system description including its performance is given in [12]. The transmission system consists of autarkic transmitter and receiver units. A subharmonic mixer is used to upconvert a signal (DC-10 GHz, delivered by an arbitrary signal source) up to $300 \mathrm{GHz}$. The transmission is performed with horn antennas directly attached to the mixer blocks. In the original setup, two dielectric phase-locked resonator oscillators are used. In this experiment, they are replaced by two commercial microwave generators phase locked to a laboratory $10-\mathrm{MHz}$ reference clock. The measurement setup is depicted in Fig. 2. The downconverted signal at the output of the RX subharmonic mixer is captured using the VSA (baseband bandwidth $40 \mathrm{MHz}$ ) or the DRTO (RF bandwidth $2.5 \mathrm{GHz}$ ). The EVM was determined by (a) proprietary software in the VSA (sampling at the VSA intermediate frequency $96 \mathrm{MHz}$ ), (b) proprietary software in the DRTO (sampling at the downconverted frequency $f_{32}$ ) and (c) in-house code (both baseband and RF samples allowed). The reason for developing the in-house code was a need for estimating uncertainties arising from EVM evaluation, which is not possible with a proprietary VSA or DRTO software. The in-house software is freely available [25] and will be further improved.

The instruments are triggered by a marker signal derived from the modulated signal symbol rate and period. In Fig. 2, $f_{31}$ is the the carrier frequency of the modulated signal and $f_{41}=\left|2 f_{21} \pm f_{31}\right|$. Similarly, $f_{42}$ is the received signal frequency spectrum and $f_{32}$ is the actual downconverted modulated signal. The power level at the receiver subharmonic mixer output is dependent on the power level at the transmitter mixer, the distance between antennas (free space loss), the gain of the antennas and the noise figure of the transmission system components. As shown in [12], the maximum achievable power level at the receiver mixer varies linearly from approximately $-100 \mathrm{dBm}$ (TX mixer level $-60 \mathrm{dBm}$ ) to approximately $-40 \mathrm{dBm}$ (TX mixer

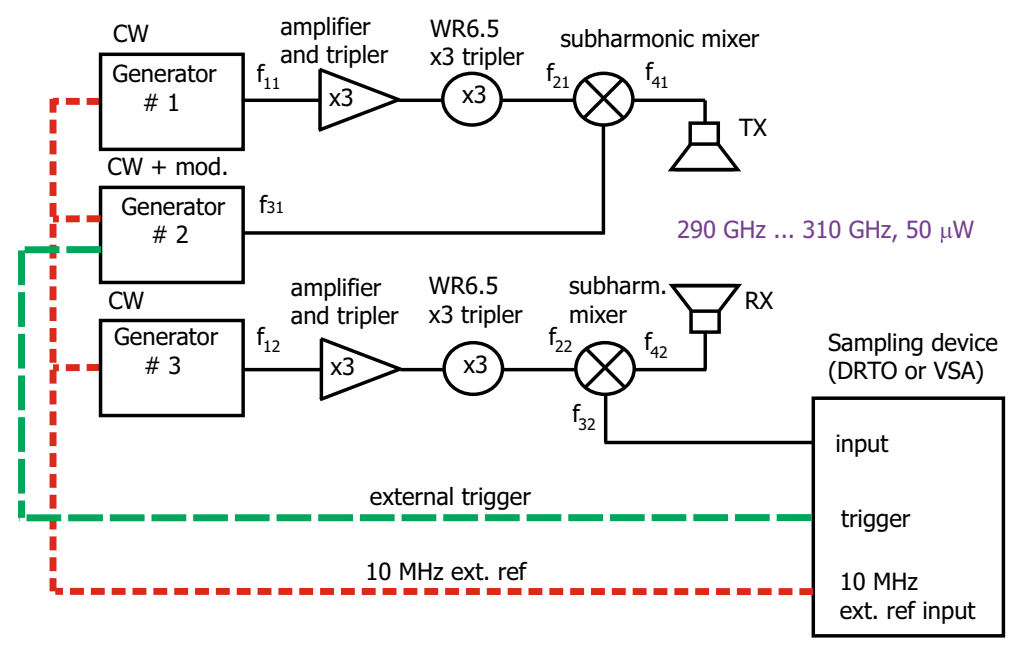

Fig. 2 Measurement setup for the measurement of modulation error parameters in the $300-\mathrm{GHz}$ transmission system 
level $-5 \mathrm{dBm}$ ). The specified gain of both RX and TX antennas is $26 \mathrm{~dB}$, respectively. The distance between the TX and RX antenna in experiments was mostly small (see Section 4) since generator \#2 is used for triggering and it is convenient to place the whole measurement setup on a single bench.

\section{Experimental Results}

The aim of the measurements was to determine the EVM of a signal at the output of the RX subharmonic mixer, i.e. the influence of the transmission system on the quality of the modulated signal. The measurement using a DRTO is more open to error analysis and consequent uncertainty calculation, whereas the VSA measurements are much faster and more easy to perform. The signal quality of the vector signal generator (VSG) itself was studied separately. The DRTO measurements were performed for a limited number of configurations, only. The measurement uncertainty is given for DRTO measurements, only.

First, the EVM of a 16QAM signal without using the $300-\mathrm{GHz}$ transmission system (in the following text referred to as "TX system") with carrier frequency of $100 \mathrm{MHz}$ was determined using three different methods to prove their agreement as mentioned in Section 3. The comparison given in Table 1 shows a good agreement among the methods, and thus, for the overall testing, EVM calculated by the VSA was used. For the uncertainty calculation example, the DRTO measurements were used and the EVM was evaluated using the in-house code [25]. It is expected that the TX system impairments remain unchanged in both cases, only the uncertainty of the signal capture and uncertainty of the EVM evaluation differ.

\subsection{Transmission of Non-distorted Signals (Vector Signal Analyzer Measurements)}

The "non-distorted" signal is here referred to as the modulated signal from the VSG without any artificial distortions introduced. Where appropriate, a comparison between the EVM of the modulated generator itself (in figures referred to as "gen. only") and the EVM of the signal at the receiver subharmonic mixer output (in figures

Table 1 EVM calculated using three different methods

\begin{tabular}{llll}
\hline $\begin{array}{l}\text { Symbol rate } \\
(\mathrm{MS} / \mathrm{s})\end{array}$ & $\begin{array}{l}\text { In-house } \\
\text { code }\end{array}$ & $\begin{array}{l}\text { DRTO } \\
\text { software }\end{array}$ & $\begin{array}{l}\text { VSA } \\
\text { software }\end{array}$ \\
\hline 0.2 & 0.19 & 0.18 & 0.19 \\
0.5 & 0.21 & 0.20 & 0.21 \\
1 & 0.25 & 0.26 & 0.22 \\
2 & 0.36 & 0.35 & 0.24 \\
5 & 0.59 & 0.60 & 0.41 \\
10 & 1.17 & 1.12 & 1.03 \\
20 & 2.27 & 2.17 & 2.28 \\
\hline
\end{tabular}




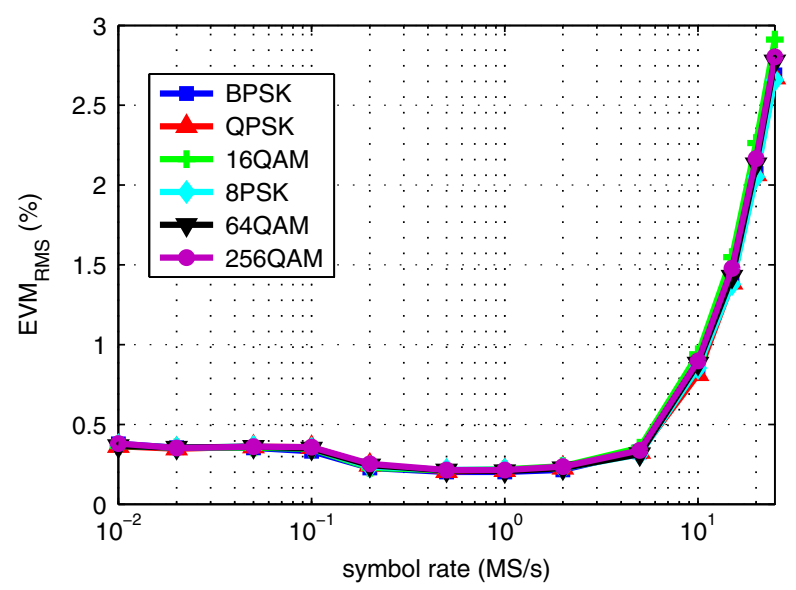

Fig. 3 EVM of the modulated generator only (direct connection of the generator and VSA through a cable with matching attenuator); generator output level was approximately $-10 \mathrm{dBm}$, average from 20 measurements (standard deviation $0.01 \div 0.02 \%$ )

referred to as "TX system") will be carried out. The EVM in this subsection was determined using the proprietary software installed in the VSA.

First, the EVM of the generator itself with different modulation schemes was measured. The symbol rate was $10 \mathrm{kS} / \mathrm{s}$ to $25 \mathrm{MS} / \mathrm{s}$, whereas the generator output level was approximately $-10 \mathrm{dBm}$ (see Fig. 3). The EVM reading may be influenced by the VSA input level setting. It is important to keep the $1^{\text {st }}$ mixer level within bounds specified by the VSA manufacturer. Improper combination of the input attenuator and reference level setting may increase the noise floor or cause the $1^{\text {st }}$ mixer overdrive. In both cases, the measured EVM value is increased due to measurement equipment imperfections, not due to the signal distortion. EVM measured for different signal levels with correct setting of the VSA $1^{\text {st }}$ mixer level is shown in Fig. 4 (the level

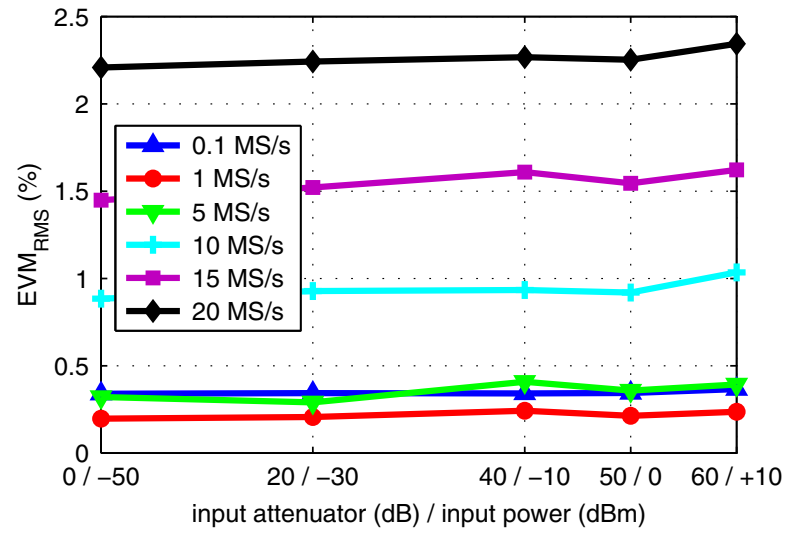

Fig. 4 EVM reading of the VSA for different power levels at the input connector with constant amplitude at the $1^{\text {st }}$ mixer. 16QAM modulated signal, carrier frequency $1 \mathrm{GHz}$, measurement without the TX system, average from 20 measurements 
of approximately $-50 \mathrm{dBm}(\mathrm{RMS})$ at the receiver subharmonic mixer output is still suitable for EVM measurements using the VSA).

After transmission of the signal using the TX system, the signal level at the input of the VSA is approximately $-50 \mathrm{dBm}$ RMS (corresponding peak envelope power $\approx-32 \mathrm{dBm}$ ) and the resulting EVM is shown in Fig. 5. The distance between RX and TX antennas was $2 \mathrm{~mm}$. It can be seen that transmission of the signal with different modulation schemes influences the quality of the signal similarly. Compared to Fig. 3, the EVM dependence on the symbol rate is masked by the EVM which is added by the transmission system. The higher EVM for low symbol rates is caused by the higher phase instability after the frequency upconversion (see Fig. 2). Signals with low symbol rates need more time for acquisition compared to signals with higher symbol rates. The system frequencies were $f_{11}=16.665 \mathrm{GHz}, f_{31}=814 \mathrm{MHz}$ and $f_{12}=16.713 \mathrm{GHz}$, which corresponds to $f_{32}=50$ and $1678 \mathrm{MHz}$, whereas only the signal component with frequency $f_{32}=1678 \mathrm{MHz}$ was used for further analysis.

The EVM was calculated from 511 symbols, which is equal to 1 period of a PN9 pseudorandom bit sequence with length of 511 bits. The number of symbols from which the EVM is calculated is usually recommended for particular communication standards [21], and generally, the EVM increases with the number of symbols as the phase errors are more difficult to compensate for long signal epochs (see Fig. 6).

\subsection{Transmission of Distorted Signals (Vector Signal Analyzer Measurements)}

In order to test the nonlinear behaviour of the transmission system, modulated signals with various impairments have been transmitted. Again, EVM of the generator itself and then EVM of the signal at the output of the RX subharmonic mixer were measured. The distance between RX and TX antennas was $2 \mathrm{~mm}$. Two different distorted signals were transmitted. First, inphase (I) and quadrature (Q) component

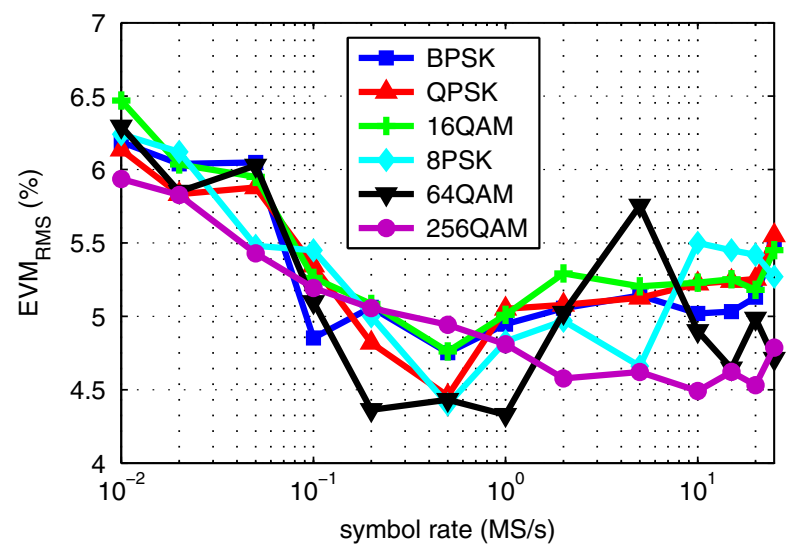

Fig. 5 EVM of the signal at the output of the receiver subharmonic mixer. Distance of the antennas $2 \mathrm{~mm}$, signal level at the VSA input was approximately $-50 \mathrm{dBm}$, average from 20 measurements (standard deviation $0.3 \div 0.4 \%$ ) 


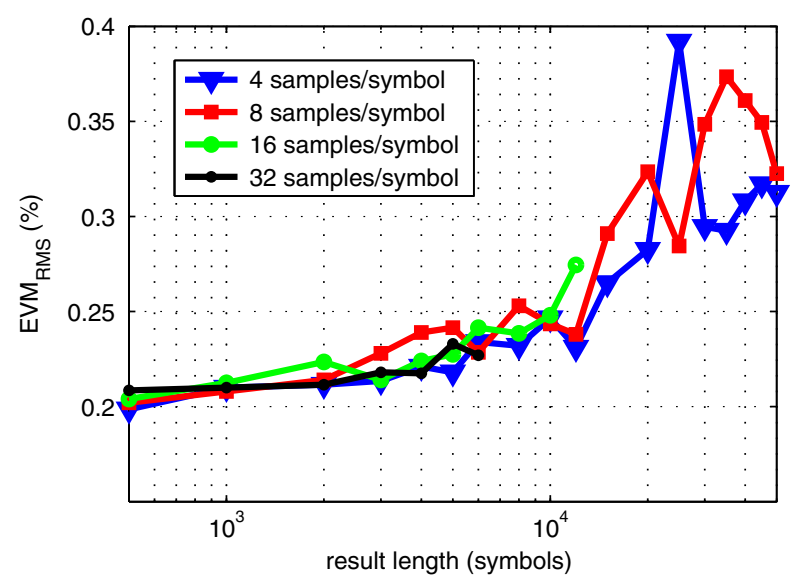

Fig. 6 EVM value in dependence on the analyzed epoch length. Generator only, QPSK modulation, carrier frequency $1 \mathrm{GHz}$, symbol rate $1 \mathrm{MS} / \mathrm{s}$, different number of samples per symbol. Each EVM value corresponds to average from 20 measurements

offsets from $0^{\circ}$ to $6^{\circ}$ were set in the generator. With a quadrature offset, the phase angle between the I and Q vector deviates from the ideal $90^{\circ}$, whereas the amplitudes of both components are of the same size. This imperfection causes the constellation diagram to flatten. The I and Q offset generally adds a DC bias to each of the I and $\mathrm{Q}$ components. At the input of the receiver, this offset has to be compensated prior to calculating EVM [21]. The quadrature offset causes a constant increase of the EVM value for all sample rates (EVM of the generator, only), whereas the signal at the output of the TX system is more influenced for symbol rates below $1 \mathrm{MS} / \mathrm{s}$ (see Fig. 7). Interestingly, the TX system slightly improves the IQ offset, as seen from Fig. 8. The

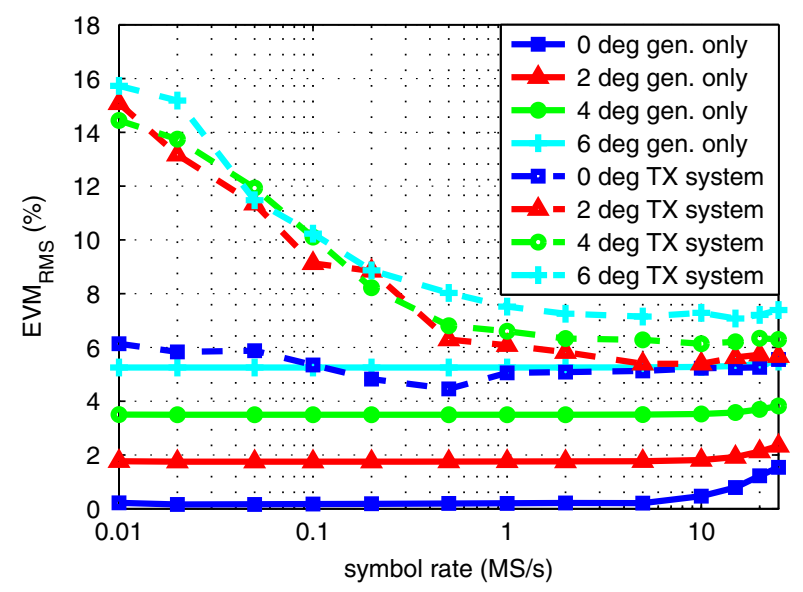

Fig. 7 Comparison of the EVM of the ideal signal from the generator (solid line) and the same signal at the output of the TX system (dashed line). Symbol rates from $10 \mathrm{kS} / \mathrm{s}$ up to $25 \mathrm{MS} / \mathrm{s}$ and QPSK modulation. The effect of quadrature error can be seen 


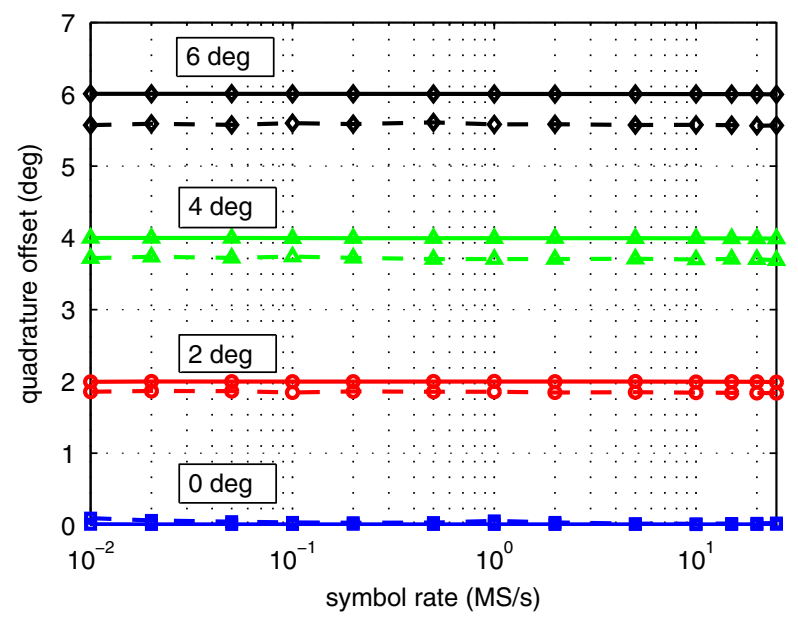

Fig. 8 Comparison of the quadrature offset measured by the VSA for both generator, only (solid line), and TX system (dashed line). The quadrature offset set at the generator is indicated above the lines. QPSK modulation is used

IQ offset value was measured using a built-in function in the VSA software both for the generator itself and for the signal transmitted using the TX system.

Second, the IQ imbalance from 0 to $0.6 \mathrm{~dB}$ was set at the generator. The imbalance causes different gain of both components and thus distortion of the constellation diagram. As seen from Fig. 9, the IQ imbalance systematically increases the EVM for all measured symbol rates, whereas the TX system adds additional distortion for symbol rates below $1 \mathrm{MS} / \mathrm{s}$. In Fig. 10, the IQ imbalance measured by an internal

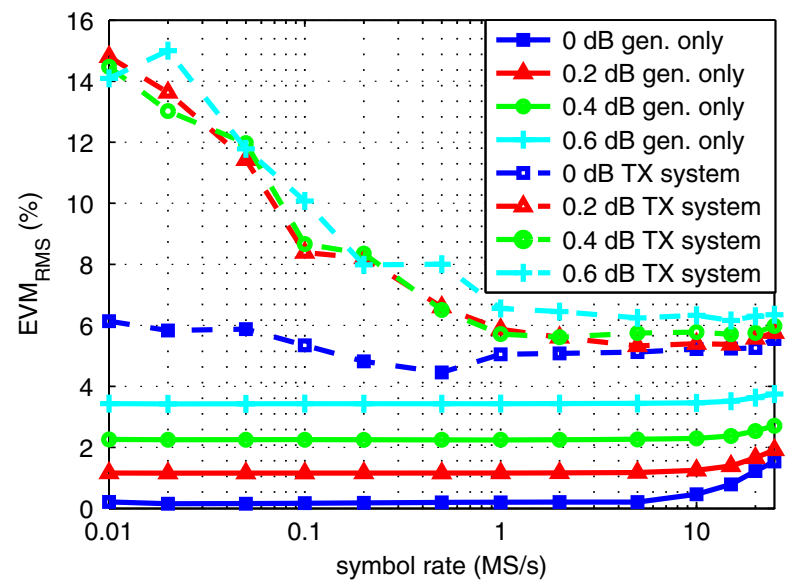

Fig. 9 Comparison of the EVM of the signal from the generator (solid line) and the same signal at the output of the TX system (dashed line). Symbol rates from $10 \mathrm{kS} / \mathrm{s}$ up to $25 \mathrm{MS} / \mathrm{s}$ using QPSK modulation. The effect of IQ imbalance can be seen 


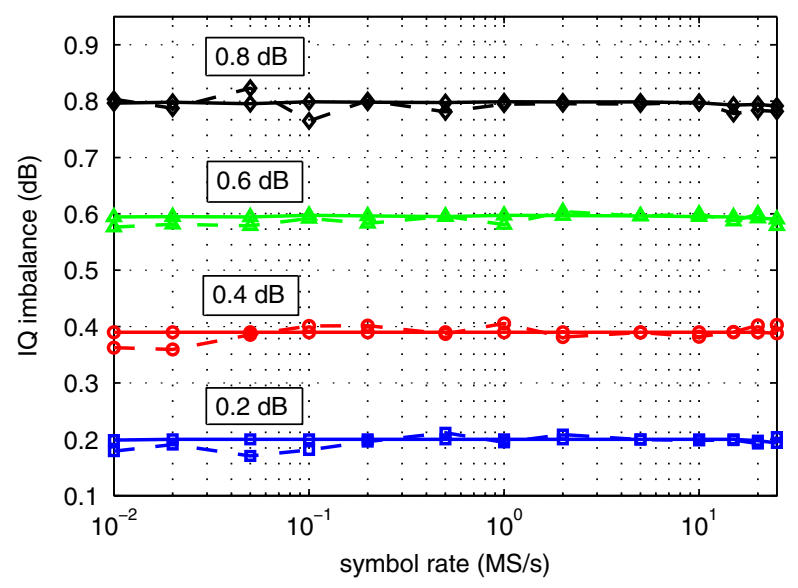

Fig. 10 Comparison of the IQ imbalance measured by the VSA for both generator only (solid line) and TX system (dashed line). The IQ imbalance set at the generator is indicated above the lines. QPSK modulation is used

function of the VSA software is plotted. It can be seen that the 300-GHz transmission system has almost no effect on the resulting signal IQ imbalance.

\subsection{Transmission of Non-distorted Signals (Digital Real Time Oscilloscope Measurements)}

The main difference between measurements using a VSA and this experiment is that the signal is sampled directly at the RF frequency and not in the baseband, i.e. the RF frequency of generator \# 2 is generally transformed into another frequency band at the output of the RX mixer. The generator \#2 RF frequency would be referred to as a carrier frequency in a "standard" transmission system, whereas here it becomes the intermediate frequency of the $300-\mathrm{GHz}$ transmission system. The downconversion of the signal into the baseband is then performed mathematically in a computer. The signal is heavily oversampled with hundreds to thousands samples per symbol in contrast to 4 to 32 samples per symbol in a VSA. The sample rate of the oscilloscope and acquisition length were set in order to obtain 1000 symbols for each measured signal. An auxiliary RF generator was added to the measurement setup for correction of the oscilloscope time base [17].

An experiment was performed with system frequencies $f_{11}=16.676 \mathrm{GHz}, f_{31}$ $=130 \mathrm{MHz}$ and $f_{12}=16.688 \mathrm{GHz}$, which corresponds to $f_{32}=86$ and $346 \mathrm{MHz}$. Only the signal with frequency $f_{32}=86 \mathrm{MHz}$ was evaluated and other components were filtered. A 16QAM signal was generated, filtered using a root-raised cosine filter (roll-off factor 0.35) and transmitted using the TX system. The distance between antennas was $20 \mathrm{~mm}$. EVM of the same signal was measured again without the transmission system and both results were compared. The comparison between EVM with and without the transmission system is shown in Fig. 11 (averaging 64 of the 


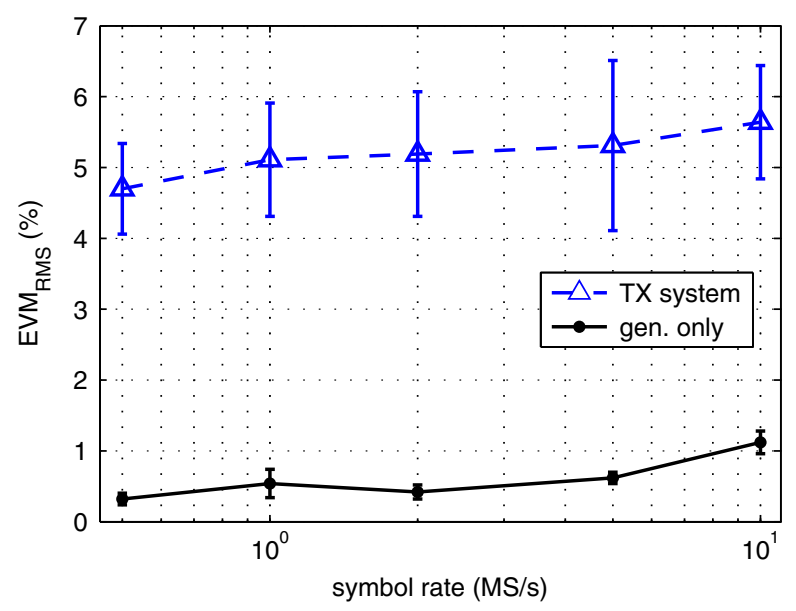

Fig. 11 Comparison of EVM measurement performed both with and without the $300-\mathrm{GHz}$ transmission system

measured time traces). The error bars correspond to the expanded uncertainty (confidence level 95\%). The averaging significantly improves the measurement dynamic range together with filtering of unwanted spectral components. The theoretical limit of the signal-to-noise ratio (SNR) of a general analog-to-digital converter (ADC) is based on the quantization noise due to the quantization error inherent in the analogto-digital conversion process when there is no oversampling and averaging. The best case SNR in decibels is calculated as a function of the effective number of bits (ENOB) of a data conversion as [26] SNR $=6.02 \cdot$ ENOB +1.76 , which gives $49.92 \mathrm{~dB}$ for 8 bits of vertical resolution (standard resolution specified by the manufacturer of the DRTO) or $67.98 \mathrm{~dB}$ for 11 bits of vertical resolution (value specified by the manufacturer of the DRTO with use of averaging). For a $1 \%$ EVM, the SNR is approximately $40 \mathrm{~dB}$ [27].

\section{EVM Measurement Uncertainty}

In metrology, a measured value is only complete if it is accompanied by a statement of the associated uncertainty (for more information, see, e.g. [28]). The uncertainty is a quantitative representation of the measurement process quality and consists of type $\mathrm{A}$ and type $\mathrm{B}$ contributions. Type $\mathrm{A}$ method of evaluation of uncertainty $u_{\mathrm{A}}$ is a statistical analysis of series of observations and type B is evaluation of uncertainty $u_{\mathrm{B}}$ by means other than the statistical analysis of series of observations. For a more realistic uncertainty estimate, an expanded uncertainty of measurement is used, obtained by multiplying the standard uncertainty $u(y)$ by a coverage factor $k$, which may be different for various statistical distributions attributed to the output estimate (for details, see [28]). For coverage probability of 95\%, the standard coverage factor $k=2$ is used. 
The EVM uncertainty evaluation is a complicated process with many unknowns. As mentioned in Section 4, the uncertainty analysis will be carried out for oscilloscope measurement as it is more open to error analysis in comparison with the VSA. The generator together with the TX system are considered to be a black box, of which EVM is determined. Separating the EVM influence of the source and receiver poses a difficult problem (for details see [17]).

\subsection{Uncertainty of the Oscilloscope Measurement}

The uncertainty of oscilloscope measurements is usually determined for single parameters, such as amplitude and rise time, and recommendations exist for evaluation of main uncertainty contributions (see, e.g. [29] and references therein). In our case, however, the whole oscilloscope waveform is stored which comprises thousands to millions of samples and the waveform metrology approach must be used $[15,16$, 18]. The TX system operating frequencies have been selected such that they minimize the influence of DRTO impairments. The error vector power (EVP) approach has been adopted [17], and the main EVM measurement uncertainty contributions are the impedance match correction, RF flatness correction, residual random component and the noise contribution.

\subsection{Uncertainty of the Signal Processing}

The oscilloscope imperfections apply to the RF signal measurement and the timing and amplitude uncertainty of measured samples can be determined. In the case of baseband measurements, the uncertainty applies directly to the I and Q components. The uncertainty given by the determination of the optimal sample within one symbol is negligible provided a large number of samples per symbol $(\geq 200)$ is used. In commercial systems, signal re-sampling is used which artificially increases the number of samples per symbol.

One of the influences difficult to take into account properly is the signal filtering and the determination of scaling constants for EVM calculation. For each modulation type, scaling constants are calculated which correspond to the symbol energy both for measured and ideal constellations [24]. This approach requires uniform statistical distribution of all received symbols.

\subsection{Uncertainty Budget Example}

An example of uncertainty calculation is summarized in Table 2. The example corresponds to the EVM calculated using the in-house code, 16QAM modulation, symbol rate $1 \mathrm{MS} / \mathrm{s}$, without averaging at the oscilloscope. The EVM was determined from ten measurements (each measurement consists of analysis of 64 oscilloscope traces), leading to the type A uncertainty of $0.36 \%$. The effective number of bits of the oscilloscope was $N=10$ (determined from the SNR [26]), the vertical resolution was $5 \mathrm{mV} /$ div, and the peak-to-peak amplitude of the signal was approximately $40 \mathrm{mV}$, which leads to the uncertainty of $0.04 \%$ in the third row in Table 2 . The specified time base accuracy is $\pm 2.5 \%$ (significantly improved by using the auxiliary RF 
Table 2 Example of EVM measurement uncertainty budget

\begin{tabular}{lll}
\hline Component & $\begin{array}{l}\text { Probability } \\
\text { distribution }\end{array}$ & $\begin{array}{l}\text { Uncertainty } \\
\text { contrib. } u_{i}(y)(\%)\end{array}$ \\
\hline $\begin{array}{l}\text { Impedance } \\
\text { mismatch }\end{array}$ & U-shaped & 0.04 \\
RF flatness & Rectangular & 0.06 \\
$\begin{array}{l}\text { Resolution } \\
\text { Optimal }\end{array}$ & Rectangular & 0.04 \\
sample & Rectangular & 0.02 \\
Random & Gaussian & 0.11 \\
noise & Rectangular & 0.01 \\
Time base & & \\
correction & Rectangular & 0.03 \\
Filtering & Rectangular & 0.05 \\
Amplitude & & \\
scaling & Gaussian & 0.36 \\
Type A & &
\end{tabular}

generator, see [17]). Uncertainty of determination of an optimal sample within one symbol and filtering was determined by simulation. All uncertainties are assumed to be uncorrelated with sensitivity coefficient equal to 1 [28]. The result is $\mathrm{EVM}_{\mathrm{RMS}}=$ $(5.11 \pm 0.78) \%$ for coverage factor $k=2$. The manufacturer of the generator does not specify the accuracy of the internal baseband generator.

\section{Conclusion}

In the paper, EVM results have been shown for a $300-\mathrm{GHz}$ transmission system for several modulation schemes and signal impairments. From the experimental results, it is obvious that the $300-\mathrm{GHz}$ transmission system influences the quality of the signal. A difference between the EVM measured directly at the output of the generator and at the output of the receiver subharmonic mixer was observed. The source of this difference, however, was not the aim of this paper. The system is able to transmit signals with both simple phase modulations (BPSK, QPSK, 8PSK) and more complicated schemes combining amplitude and phase modulation such as 16QAM, 64QAM or 256QAM. Similar distortion has been observed for all studied modulation schemes. The phase stability of the whole system is only secured when all the generators use a common frequency reference. The system is able to transmit signals with bandwidths up to $10 \mathrm{GHz}$, yet in this work, signals with much lower bandwidths were transmitted. The bandwidth of state-of-the-art VSAs is not sufficient to analyze signal bandwidths which can be transmitted using the $300-\mathrm{GHz}$ system. Besides the VSA measurements, a method using a real-time oscilloscope and consecutive signal processing in a computer has been shown, which not only is more open to measurement uncertainty analysis but also allows to measure modulated signals with bandwidths 
exceeding those of modern VSAs. The phase instability of the received signal due to frequency multiplying and mixing may be significant, which could cause problems especially for modern multicarrier modulation schemes. The influence of the channel, which also significantly contributes to the signal distortion, was not studied in this work.

Acknowledgements This work has been conducted within the EMRP joint research project "IND16 Metrology for ultrafast electronics and high-speed communications". The EMRP is jointly funded by the EMRP-participating countries within EURAMET and the European Union.

\section{Compliance with Ethical Standards}

Conflict of interests The authors declare that they have no conflict of interest.

Open Access This article is distributed under the terms of the Creative Commons Attribution 4.0 International License (http://creativecommons.org/licenses/by/4.0/), which permits unrestricted use, distribution, and reproduction in any medium, provided you give appropriate credit to the original author(s) and the source, provide a link to the Creative Commons license, and indicate if changes were made.

\section{References}

1. K. Huang and Z. Wang, Millimeter Wave Communication Systems, ser. IEEE Series on Digital \& Mobile Communication. Hoboken: Wiley-IEEE Press, 2001.

2. R. C. Daniels, J. N. Murdock, T. S. Rappaport, and R. W. Heath. "60 GHz wireless: Up close and personal,” Suppl. to IEEE Microwave Mag., vol. 11, no. 7, pp. S44-S50, 2010.

3. S. Horiguchi, K. Arakawa, Y. Minamikata, and T. Nagatsuma. "Error-free 30-50 Gbps wireless transmission at $300 \mathrm{GHz}$," in Proc. of Asia-Pacific Microw. Conf., Seoul, Korea, Nov. 2013, pp. 660-662.

4. R. Piesiewicz, T. Kleine-Ostmann, N. Krumbholz, D. Mittleman, M. Koch, J. Schoebel, and T. Kürner. "Short-range ultra-broadband terahertz communications: Concepts and perspectives," IEEE Antennas Propagat. Mag., vol. 49, no. 6. pp. 24-39, 2007.

5. J. Federici and L. Moeller. "Review of terahertz and subterahertz wireless communications," Appl. Phys. Lett., vol. 107, p 111101, 2010.

6. D. Grischkowsky. "Digital THz communication links in the atmosphere," in 38th International Conf. on Infrared, Millimeter, and Terahertz Waves (IRMMW-THz), Mainz, Germany, Sep. 2013, pp. 1-2.

7. J.-H. Seo and W.-Y. Choi. "Gigabit transmission in 60-GHz-band using optical frequency upconversion by semiconductor optical amplifier and photodiode configuration," in IEEE MTT-S International Microwave Symposium Digest, San Francisco, USA, Jun. 2006, pp. 22-25.

8. A. Hirata, J. Takeuchi, D. Kim, and J. Hirokawa. "10-Gbit/s dual channel transmission of 120GHz-band wireless link using planar slot array antennas," in Proc. of the $43^{\text {rd }}$ European Microwave Conference, Nuremberg, Germany, Oct. 2013, pp. 744-747.

9. M. Tytgat, M. Steyaert, and P. Reynaert. "Generation of Gbit/s modulated millimeter wave signals for measurement," in Proc. of the $41^{s} t$ European Microwave Conference, Manchester, UK, Oct. 2011, pp. 906-909.

10. S. Koenig, F. Boes, D. Lopez-Diaz, J. Antes, R. Henneberger, R. Schmogrow, D. Hillerkuss, R. Palmer, T. Zwick, C. Koos, W. Freude, O. Ambacher, I. Kallfass, and J. Leuthold. "100 Gbit/s wireless link with mm-wave photonics," in Optical Fiber Communication Conference and Exposition and the National Fiber Optic Engineers Conference (OFC/NFOEC), Annaheim, CA, USA, Mar. 2013, pp. 1-3. 
11. G. Ducornau, P. Szriftgiser, F. Pavanello, P. Latzel, A. Beck, T. Akalin, E. Peytavit, M. Zaknoune, D. Bacquet, and J. F. Lampin. "22 Gbps wireless communication system at $0.4 \mathrm{THz}$ ", in 38th International Conf. on Infrared, Millimeter, and Terahertz Waves (IRMMW-THz), Mainz, Germany, Sep. 2013, pp. 1-2.

12. C. Jastrow, K. Münter, R. Piesiewicz, T. Kürner, M. Koch, and T. Kleine-Ostmann. " $300 \mathrm{GHz}$ transmission system," Electronics Lett., vol. 44, no. 3, pp. 213-214, 2008.

13. C. Jastrow, S. Priebe, B. Spitschan, J. Hartmann, M. Jacob, T. Kürner, T. Schrader, and T. Kleine-Ostmann. "Wireless digital data transmission at $300 \mathrm{GHz}$," Electronics Lett., vol. 46, no. 9 , pp. 661-663, 2010.

14. D. A. Humphreys and J. Miall. "Traceable RF peak power measurements for mobile communications," IEEE Trans. Instrum. Meas., vol. 54, no. 2, pp. 680-683, 2005.

15. D. A. Humphreys and R. T. Dickerson. "Traceable measurement of error vector magnitude (EVM) in WCDMA signals," in Proc. of 2007 Internat. Waveform Diversity \& Design Conf. Pisa, Italy, Jun. 2007, pp. 270-274.

16. D. A. Humphreys, M. R. Harper, L. K. J. McInnes, and J. Miall. "Strategy for traceability of complex modulated signals using RF waveform metrology," in Proc. of XXIX URSI General Assembly, Chicago, USA, Aug. 2008, paper A03.6.

17. D. A. Humphreys and J. Miall. "Traceable measurement of source and receiver EVM using a real-time oscilloscope," IEEE Trans. Instrum. Meas., vol. 62, no. 6, pp. 1413-1416, 2013.

18. D. A. Humphreys, M. Hudlička, and I. Fatadin. "Calibration of Wideband Digital Real-Time Oscilloscopes,” IEEE Trans. Instrum. Meas., vol. 64, no. 6, pp. 1716-1725, 2015.

19. G. Jue and T. Dippon. "Addressing the challenges of wideband waveform generation and analysis," Microwave Journal, vol. 54, no. 8, pp. 20-24, 2011.

20. M. Hudlička, C. Jastrow, T. Schrader, and T. Kleine-Ostmann. "Waveform metrology for error vector magnitude measurements in a $300 \mathrm{GHz}$ transmission system," in 2012 Conference on Precision Electromagnetic Measurements, Washington, USA, Jul. 2012, pp. 526-527.

21. 3rd Generation Partnership Project, "Technical specification group radio access network; base station (BS) conformance testing (FDD)," 3GPP TS 25.141, version 9.5.0 Release 9, 2010-09.

22. T. L. Jensen and T. Larsen. "Robust computation of error vector magnitude for wireless standards," IEEE Trans. Commun., vol. 61, no. 2, pp. 648-657, 2013.

23. Rumney, M. Ed., LTE and the Evolution to $4 G$ Wireless: Design and Measurement Challenges, 2nd ed. John Wiley \& Sons Inc, 2013.

24. M. D. McKinley, K. A. Remley, M. Myslinski, J. S. Kenney, D. Schreurs, and B. Nauwelaers, "EVM calculation for broadband modulated signals", in 64th ARFTG Conf. Dig., Orlando, FL, Dec. 2004, pp. 45-52.

25. "Metrology for ultrafast electronics and high-speed communications," EMRP joint research project. [Online]. Available: http://www.ptb.de/emrp/ultrafast.html.

26. W. R. Bennett. "Spectra of quantized signals," Bell System Technical Journal, vol. 27, pp. 446-471, 1948.

27. H. A. Mahmoud. "Error vector magnitude to SNR conversion for nondata-aided receivers," IEEE Trans. Wireless Commun., vol. 8, no. 5, pp. 2694-2704, 2009.

28. Expression of the Uncertainty of Measurement in Calibration EA-4/02, European co-operation for Accreditation Std., 1999. [Online]. Available: http://www.european-accreditation.org.

29. Calibration of Measuring Devices for Electrical Quantities: Calibration of Oscilloscopes, EURAMET e.V., Technical Committee for Electricity and Magnetism Std., 2011. [Online]. Available: http://www.euramet.org/. 